\title{
Comparative flavan-3-ol composition of seeds from different grape varieties
}

\author{
C. Santos-Buelga, E. M. Francia-Aricha \& M. T. Escribano-Bailon \\ Departamento de Química Analitica, Nutrición y Bromatologia, Universidad de Salamanca. Avda. Campo Charro s/n. \\ 37007-Salamanca, Spain
}

(Received 18 May 1994; revised version received and accepted 15 October 1994)

\begin{abstract}
The flavanol composition of the seeds of 17 varieties of grape cultivated in the main wine-producing areas of Castilla-Leon (Spain) was studied. Twenty-seven different flavan-3-ols of procyanidin type were found, but no prodelphinidins were detected. Minor amounts of four hydrolyzable tannins were also present in the seed extracts of most of the grapes analyzed. All the varieties contained galloyled flavanols, such that their presence could be considered characteristic of the grape seed composition. Some proposals concerning the biosynthesis of flavan3-ol in the grape seed are also advanced taking into account the compounds found.
\end{abstract}

\section{INTRODUCTION}

The presence and distribution of proanthocyanidins in grape seeds have been studied by several authors (Bourzeix et al., 1986; Lea et al., 1979; Oszmianski \& Sapis, 1989; Da Silva et al., 1991 $a, 1991 b$ and 1992; Romeyer et al., 1986). The compounds characterized are present in the soluble fraction since an as yet poorly quantified part of them is bound to the matrix polysaccharides (Haslam, 1989) and cannot be extracted without modifying their characteristics. The monomers are the most abundant flavan-3-ols in the soluble fraction and are mainly represented by $(+)$-catechin and (-)-epicatechin, as well as (-)-epicatechin-3-Ogallate. Among the oligomers, dimers are usually the most abundant compounds, the dimer B2 being the major component in seeds (Bourzeix et al., 1986; Da Silva et al., 1991 $a$ and 1992). Another characteristic of the flavanol composition of grape seeds is the presence of derivatives esterified with gallic acid (Da Silva et al., 1991a, 1992).

Most stages in the synthesis of flavan-3-ols are known, although some steps have not been well established, such as the pathway for the formation of derivatives with absolute $3 R$ stereochemistry ((-)-epicatechin type) and the pathway for the condensation that gives rise to oligomer proanthocyanidins (in this respect, see reviews by Heller \& Forkman, 1993 and Stafford, 1989, 1993). In the present work we studied the flavan-3-ol compounds present in grape seeds in order to draw some general conclusions about the flavanol composition and the synthesis of these types of compounds.

\section{MATERIALS AND METHODS}

\section{Samples}

A total of 27 samples of seeds from the grapes of 17 different varieties of Vitis vinifera ( 7 white and 10 red) collected during the 1992 harvest in different areas of the community of Castilla-Leon (Spain) was analyzed. The grapes were collected when enologically ripe and the seeds were removed manually and kept at $-40^{\circ} \mathrm{C}$ until analysis.

\section{Extraction of flavan-3-ol}

From 1 to $2 \mathrm{~g}$ of frozen seeds were ground and repeatedly homogenized in methanol containing $0.5 \mathrm{~g} /$ litre of ascorbic acid. A volume of water was then added to this and the mixture was evaporated under vacuum until the methanol had been removed. The aqueous extract was washed with $n$-hexane in order to remove liposoluble substances and the remaining solvent was removed by evaporation. The final aqueous extract was evaporated under vacuum to a volume of 3-5 ml, filtered through a $0.45 \mu \mathrm{m}$ filter and analyzed by high performance liquid chromatography (HPLC).

\section{Analysis of flavan-3-ol}

This was carried out by HPLC/DAS following a previously-described method (Escribano-Bailon et al., 1992). The compounds appearing in the chromatograms were identified by comparison with standards and by techniques of hydrolysis. The latter are also reported in the above reference. 


\section{RESULTS AND DISCUSSION}

Figure 1 shows a typical HPLC chromatogram of a methanolic extract of grape seeds corresponding to a sample of the Cabernet-Sauvignon variety. The composition of the seed extracts of all the varieties analyzed was characterized for the almost exclusive presence of flavan-3-ols. Also detected were gallic acid and some compounds identified as hydrolyzable tannins (peak spectra showing a maximum wavelength at 275 $\mathrm{nm})$ since their enzymatic hydrolysis performed with tannase (TA 891005, Kikkoman) released gallic acid and glucose. Minor amounts of four substances (peaks

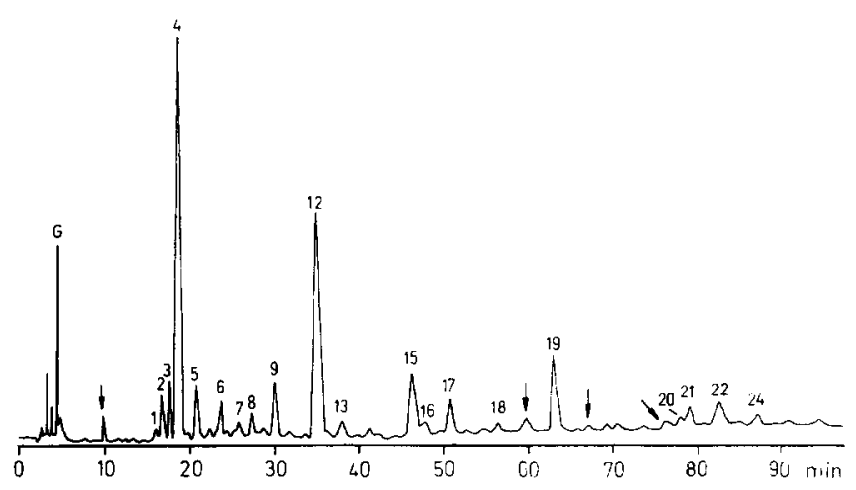

Fig. 1. HPLC chromatogram recorded at $280 \mathrm{~nm}$ of an extract of grape seeds from Vitis vinifera variety CabernetSauvignon. $\mathrm{G}=$ gallic acid. Peaks corresponding to hydrolyzable tannins are marked with an arrow. For flavan-3-ols identification (peaks numbered) see Table 1. marked with an arrow in Fig. 1) of this type were observed in the HPLC chromatograms of most of the samples analyzed.

Twenty-seven different flavan-3-ols were identified (Table 1), of which, as far as we are aware, three were detected for the first time in grape seeds. All the oligomers encountered were of the procyanidin type, no prodelphinidins or derivatives of another type being found. Proanthocyanidins esterified with gallic acid were found in the seeds of all the samples analyzed such that their presence could be considered characteristic of the flavan composition of grape seeds. Galloylation always occurred on an epicatechin unit, no compound with catechin-O-gallate being found.

Table 2 shows the mean flavanol distribution of grape seeds of the different varieties for the individually most important compounds and the set of galloylated derivatives and of dimers with $\mathrm{C} 4-\mathrm{C} 6$ linkages. The distribution percentages are given as the contribution of each peak to the sum of the total of the peak areas present in the chromatograms. In the extracts, monomers were found to be the most abundant flavan3-ol compounds (means of $40 \%$ ), $(+)$-catechin generally being more abundant than (-)-epicatechin. The proanthocyanidins present in the extracts are mainly dimers and trimers in which the elemental units are essentially bound by type $\mathrm{C} 4 \rightarrow \mathrm{C} 8$ interflavan bonds. The areas of the peaks corresponding to dimer compounds represent $28 \%$ of the overall set of flavan-3-ols, only $3 \%$ of this corresponding to derivatives with $\mathrm{C} 4 \rightarrow \mathrm{C} 6$ linkages. No procyanidins showing type A double linkages were

Table 1. Flavan-3-ol isolated from grape seeds

\begin{tabular}{|c|c|}
\hline Peak number ${ }^{a}$ & Compound \\
\hline 1 & Catechin- $(4 \alpha \rightarrow 8)$-catechin- $(4 \alpha \rightarrow 8)$-catechin $(\mathrm{C} 2)$ \\
\hline 2 & Catechin- $(4 \alpha \rightarrow 8)$-catechin (B3) \\
\hline 3 & Epicatechin- $(4 \beta \rightarrow 8)$-catechin (B1) \\
\hline 4 & $(+)$-Catechin \\
\hline 5 & Epicatechin- $(4 \beta \rightarrow 8)$-epicatechin- $(4 \beta \rightarrow 8)$-catechin \\
\hline 6 & Catechin- $(4 \alpha \rightarrow 8)$-epicatechin (B4) \\
\hline $7 \mathrm{a}$ & Catechin- $(4 \alpha \rightarrow 8)$-catechin-( $4 \alpha \rightarrow 8)$-epicatechin ${ }^{b}$ \\
\hline $7 \mathrm{~b}$ & Epicatechin- $(4 \beta \rightarrow 6)$-epicatechin- $(4 \beta \rightarrow 8)$-catechin \\
\hline $8 \mathrm{a}$ & Catechin- $(4 \alpha \rightarrow 6)$-catechin (B6) \\
\hline $8 b$ & Epicatechin- $(4 \beta \rightarrow 6)$-epicatechin- $(4 \beta \rightarrow 8)$-epicatechin \\
\hline 9 & Epicatechin- $(4 \beta \rightarrow 8)$-epicatechin (B2) \\
\hline 10 & Epicatechin- $(4 \beta \rightarrow 8)$-epicatechin-3-O-gallate- $(4 \beta \rightarrow 8)$-catechin \\
\hline 11 & Epicatechin-3-O-gallate- $(4 \beta \rightarrow 8)$-epicatechin (B2-3-O-gallate) \\
\hline 12 & (-)-Epicatechin \\
\hline 13 & Catechin- $(4 \alpha \rightarrow 8)$-epicatechin-3-O-gallate (B4-3'-O-gallate) \\
\hline 14 & Epicatechin- $(4 \beta \rightarrow 8)$-epicatechin- $(4 \beta \rightarrow 6)$-catechin \\
\hline $15 \mathrm{a}$ & Epicatechin-3-O-gallate-( $4 \beta \rightarrow 8)$-catechin (B1-3-O-gallate) \\
\hline $15 \mathrm{~b}$ & Epicatechin- $(4 \beta \rightarrow 8)$-epicatechin-3-O-gallate (B2-3'-O-gallate) \\
\hline 16 & Epicatechin-(4B $\rightarrow 6)$-catechin (B7) \\
\hline 17 & Epicatechin- $(4 \beta \rightarrow 8)$-epicatechin- $(4 \beta \rightarrow 8)$-epicatechin $(\mathrm{C} 1)$ \\
\hline 18 & Epicatechin- $(4 \beta \rightarrow 8)$-epicatechin- $(4 \beta \rightarrow 8)$-epicatechin- $(4 \beta \rightarrow 8)$-epicatechin \\
\hline 19 & (-)-Epicatechin-3-O-gallate \\
\hline 20 & Epicatechin-3-O-gallate- $(4 \beta \rightarrow 6)$-catechin $(B 7-3-O-\text { gallate })^{b}$ \\
\hline 21 & Epicatechin-3-O-gallate-( $4 \beta \rightarrow 8)$-epicatechin-3-O-gallate (B2-3,3'-O-digallate) \\
\hline 22 & Epicatechin- $(4 \beta \rightarrow 8)$-epicatechin- $(4 \beta \rightarrow 8)$-epicatechin-3-O-gallate \\
\hline 23 & Epicatechin- $(4 \beta \rightarrow 8)$-epicatechin-3-O-gallate-( $4 \beta \rightarrow 8)$-epicatechin-3-0-gallate ${ }^{b}$ \\
\hline 24 & Epicatechin- $(4 \beta \rightarrow 6)$-epicatechin (B5) \\
\hline
\end{tabular}

${ }^{a}$ Numbering of peaks according to their order of elution.

${ }^{b}$ FIavan-3-ol identified for the first time in grape seeds. 
Table 2. Distribution of fiavan-3-ol in grape seeds

\begin{tabular}{|c|c|c|c|c|c|c|c|c|c|c|c|c|}
\hline \multirow[b]{2}{*}{ Grape variety } & \multicolumn{12}{|c|}{ HPLC area percentages ${ }^{a}$} \\
\hline & $\mathrm{C}$ & $\mathrm{E}$ & EG & B1 & B2 & B3 & B4 & $\mathrm{C} 1$ & EEC & E4 & Gall. & $(4-6)$ \\
\hline Verdejo $(n=4)$ & $36 \pm 8^{b}$ & $12 \pm 5$ & $6 \pm 2$ & $2 \pm 1$ & $4 \pm 1$ & $3 \pm 1$ & $3 \pm 1$ & $2 \pm 1$ & $4 \pm 2$ & & $14 \pm 2$ & $3 \pm 1$ \\
\hline Godello $(n=1)$ & $29 \pm 1$ & $11 \pm 1$ & $5 \pm 0$ & $6 \pm 0$ & $3 \pm 0$ & $5 \pm 1$ & $3 \pm 1$ & $2 \pm 1$ & $4 \pm 0$ & & $17 \pm 3$ & $3 \pm 1$ \\
\hline Albillo $(n=1)$ & $30 \pm 1$ & $18 \pm 1$ & $5 \pm 0$ & $4 \pm 0$ & $4 \pm 0$ & $4 \pm 0$ & $3 \pm 0$ & $2 \pm 0$ & $4 \pm 1$ & $1 \pm 0$ & $11 \pm 1$ & $4 \pm 0$ \\
\hline Malvasia $(n=2)$ & $15 \pm 6$ & $15 \pm 5$ & $5 \pm 1$ & $4 \pm 1$ & $7 \pm 1$ & $3 \pm 1$ & $7 \pm 3$ & $5 \pm 1$ & $4 \pm 1$ & $2 \pm 0$ & $9 \pm 3$ & $3 \pm 1$ \\
\hline Valenciana $(n=1)$ & $22 \pm 1$ & $21 \pm 1$ & $4 \pm 0$ & $1 \pm 0$ & $9 \pm 1$ & $4 \pm 0$ & $6 \pm 0$ & $4 \pm 0$ & $3 \pm 0$ & $1 \pm 0$ & $6 \pm 1$ & $3 \pm 0$ \\
\hline Palomino $(n=1)$ & $34 \pm 4$ & $15 \pm 1$ & $2 \pm 0$ & $5 \pm 1$ & $5 \pm 0$ & $4 \pm 0$ & $3 \pm 1$ & $3 \pm 0$ & $4 \pm 0$ & & $9 \pm 1$ & $3 \pm 1$ \\
\hline Sauvignon $(n=1)$ & $38 \pm 1$ & $12 \pm 0$ & $6 \pm 1$ & $2 \pm 0$ & $3 \pm 0$ & $3 \pm 0$ & $3 \pm 0$ & $2 \pm 0$ & $3 \pm 0$ & $1 \pm 0$ & $15 \pm 1$ & $3 \pm 0$ \\
\hline Tempranillo $(n=4)$ & $27 \pm 8$ & $17 \pm 4$ & $4 \pm 1$ & $2 \pm 1$ & $6 \pm 1$ & $3 \pm 1$ & $5 \pm 2$ & $3 \pm 1$ & $3 \pm 1$ & $1 \pm 0$ & $12 \pm 4$ & $3 \pm 2$ \\
\hline Tinta del Pais $(n=3)$ & $20 \pm 2$ & $17 \pm 1$ & $3 \pm 2$ & $4 \pm 0$ & $8 \pm 2$ & $3 \pm 0$ & $7 \pm 1$ & $4 \pm 1$ & $3 \pm 1$ & $1 \pm 1$ & $11 \pm 2$ & $5 \pm 1$ \\
\hline Cabernet-Sauv. $(n=1)$ & $22 \pm 1$ & $22 \pm 2$ & $8 \pm 1$ & $4 \pm 0$ & $4 \pm 0$ & $3 \pm 0$ & $3 \pm 0$ & $3 \pm 0$ & $3 \pm 0$ & $1 \pm 0$ & $15 \pm 1$ & $3 \pm 1$ \\
\hline Garnacha $(n=2)$ & $22 \pm 1$ & $23 \pm 2$ & $4 \pm 2$ & $4 \pm 1$ & $8 \pm 0$ & $3 \pm 1$ & $5 \pm 1$ & $4 \pm 0$ & $3 \pm 1$ & $1 \pm 0$ & $5 \pm 2$ & $4 \pm 1$ \\
\hline Juan García $(n=1)$ & $12 \pm 4$ & $21 \pm 3$ & $7 \pm 1$ & $3 \pm 0$ & $9 \pm 1$ & $2 \pm 0$ & $7 \pm 1$ & $6 \pm 0$ & $3 \pm 0$ & $2 \pm 1$ & $13 \pm 2$ & $5 \pm 1$ \\
\hline Malbec $(n=1)$ & $21 \pm 1$ & $24 \pm 1$ & $8 \pm 1$ & $3 \pm 0$ & $4 \pm 0$ & $2 \pm 0$ & $3 \pm 0$ & $3 \pm 0$ & $2 \pm 0$ & $1 \pm 0$ & $15 \pm 1$ & $5 \pm 1$ \\
\hline Prieto Picudo $(n=1)$ & $27 \pm 2$ & $9 \pm 1$ & $4 \pm 0$ & $5 \pm 1$ & $6 \pm 1$ & $3 \pm 0$ & $2 \pm 0$ & $3 \pm 1$ & $6 \pm 1$ & $1 \pm 0$ & $15 \pm 1$ & $8 \pm 1$ \\
\hline Mencía $(n=1)$ & $15 \pm 1$ & $19 \pm 0$ & $6 \pm 1$ & $4 \pm 0$ & $7 \pm 1$ & $3 \pm 0$ & $4 \pm 0$ & $3 \pm 0$ & $3 \pm 0$ & $2 \pm 0$ & $15 \pm 1$ & $8 \pm 1$ \\
\hline Pinot noir $(n=1)$ & $32 \pm 1$ & $25 \pm 1$ & $1 \pm 0$ & $3 \pm 1$ & $5 \pm 0$ & $2 \pm 0$ & $3 \pm 0$ & $3 \pm 0$ & $4 \pm 0$ & $2 \pm 0$ & $8 \pm 1$ & $6 \pm 1$ \\
\hline Merlot $(n=1)$ & $33 \pm 1$ & $33 \pm 1$ & $12 \pm 0$ & $1 \pm 0$ & $2 \pm 0$ & $1 \pm 0$ & $2 \pm 0$ & $1 \pm 0$ & $1 \pm 0$ & $1 \pm 0$ & $15 \pm 1$ & $5 \pm 0$ \\
\hline
\end{tabular}

In each variety, different samples are from different growing areas. The analyses of each sample were performed in triplicate.

${ }^{a}$ Only substances with more than $1 \%$ of the area are shown.

${ }^{b}$ Mean \pm SD.

Abbreviations: $C=(+)$-catechin; $E=(-)$-epicatechin; $E G=(-)$-epicatechin-3-0-gallate; $E E C=$ epicatechin-(4 $\beta \rightarrow 8)$-epicatechin- $(4 \beta \rightarrow 8)$ catechin; E4=epicatechin tetramer; Gall.=sum of galloyl derivatives (excluding (-)-epicatechin-3-0-gallate); $(4-6)=$ sum of dimers with $(4 \rightarrow 6)$ interflavanol linkage.

observed. Individually, the B2 dimer is the most abundant procyanidin, followed by the remaining dimers with $\mathrm{C} 4 \rightarrow \mathrm{C} 8$ linkages and the trimers $\mathrm{Cl}$ and epicatechin- $(4 \beta \rightarrow 8)$-epicatechin- $(4 \beta \rightarrow 8)$-catechin. On average, the peak areas of the galloylated derivatives represent $18 \%$ of the total, the proportions of epicatechin-3-Ogallate, B2-3'-O-gallate and C1-3'-O-gallate being of importance individually.

These general lines concerning the flavanol composition persist throughout the varieties studied, although among them some differences can be seen as regards the distribution of the compounds. The most outstanding differences are those occurring in the distribution of the flavan-3-ol monomers, with varieties in which (+)catechin is clearly major (white varieties in general) and others in which the percentages of (+)-catechin and (-)epicatechin are balanced or with even a predominance of the latter (e.g. the Juan García red variety). The differences in composition observed in this and other aspects can be considered to be related to the type of variety; however, the data obtained are insufficient for conclusions to be drawn since, for this, a greater number of samples of each variety should be analyzed. It should also be taken into account that the samples analyzed were from different areas and this could also lead to differences as regards composition.

Although proanthocyanidins are usually defined as oligomers of flavan-3-ol it should be noted that in fact they are not produced as a result of the autocondensation of flavan-3-ol products but through the sequential addition of flavan-3,4-diol units, in their reactive form as carbocations (or their equivalent quinone methide) to a flavan-3-ol monomer, which acts as a terminal unit (or initiation unit) (Stafford, 1993). In our case, the unit predominating for extension in the oligomers is epicatechin; only 6 of the 24 procyanidins identified have catechin in the extension units. However, catechin is the most abundant element among the flavan-3-ol monomers and in the initiation subunits; 11 of the 24 procyanidins have catechin in the terminal unit, eight display epicatechin and five epicatechin-O-gallate. Moreover, seeds with higher percentages of monomer (-)-epicatechin also show higher relative proportions of procyanidins containing this compound in the initiation unit and, likewise, the higher relative percentages of procyanidols with $(+)$-catechin in their terminal unit are generally related to elevated proportions of this monomer. These findings support the observations of Haslam et al. (1977) and Stafford et al. (1982) to the effect that the initiation units (and hence the flavan-3-ol monomers) and those of extension come from different metabolic pools.

It is not yet well established how the condensation of the units gives rise to the formation of oligomer proanthocyanidins. Using biomimetic synthesis (Ferreira $e t$ al., 1992; Haslam, 1989) the formation of oligomers by non-enzymatic techniques has been readily achieved. However, Stafford (1989) considers that in vivo there are probably one or more condensation enzymes (proanthocyanidin synthases) for the purposes of regulation. In our case, the tendency to incorporate a particular type of unit for extension could perhaps be explained in terms of the existence of condensation enzymes that would be specific for each type of carbocation. This finding supports the fact that we did not observe any oligomer simultaneously displaying catechin and epicatechin in 
the extension units. Haslam (1989), however, throws doubt on the notion that condensation would be under direct enzymatic control, since a close correlation was found between the products formed in vivo and those obtained by biomimetic synthesis, performed exclusively under thermodynamic control. If this hypothesis is accepted, there would be two possible alternatives to account for the imbalance observed between catechin and epicatechin units between extension units and initiation units and monomers: (1) that the synthesis of monomers and oligomers would take place at different times or in different compartments, and (2) that the epicatechin cabocations show a greater tendency to become stabilized by binding to another flavan than to evolve to a monomer.

We have found no references to the synthesis of galloyl esters of flavan-3-ols. The fact that until now no galloylated precursors of procyanidins have been identified indicates that acylation occurs later than the formation of flavan-3-ol structures. In our case, galloylation always occurs on epicatechin, suggesting a great specificity for the esterifying enzymes. As may be seen in Table 1, the dimers may display gallate in any position; however, we did not find any galloylated trimer compound in their upper subunit. This suggests that esterification would preferentially occur on the monomer compounds and upper units of the preformed dimers and that the galloylated trimers would be formed by extension of the previously galloylated dimer (Fig. 2).

In the scheme shown in Fig. 2, any monomer flavan3-ol [(+)-catechin, (-)-epicatechin or (-)-epicatechin-3-0gallate] can act as an initiation unit, onto which a carbocation of catechin or epicatechin is incorporated, thus forming a dimer that may or may not be galloylated on its terminal unit. Following this, the dimer formed may become galloylated on the upper unit. In this proposal, it is assumed that esterification never occurs before incorporation of the carbocation that acts as an extension element and hence molecule growth always occurs through the incorporation of non-galloylated units. In the sequence offered in Fig. 2, the terminal trimer would have its upper unit ready to be galloylated. However, as mentioned above, we have not identified any trimer esterified on this subunit; this could be interpreted as meaning that the possibility of galloylation of the trimers is remote or impossible, probably because their size would prevent their linkage to the active site of esterifying enzymes. In support of this line of reasoning is the fact that some non-galloylated trimers are found in grape seeds in amounts similar (or even higher) to those of the major dimer compounds such that if their galloylation were possible directly, the probability of trimers esterified on any of their subunits would be similar to that of the equivalent dimers.
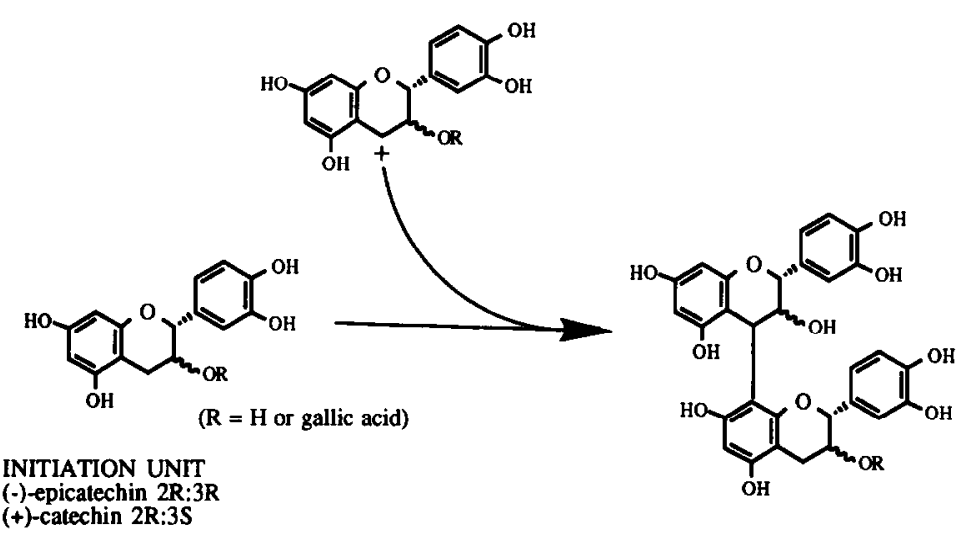
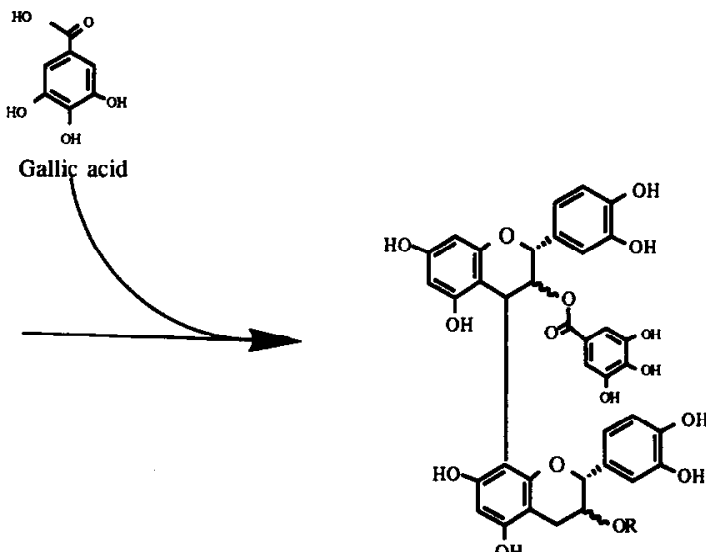

Mono- or digalloylated dime

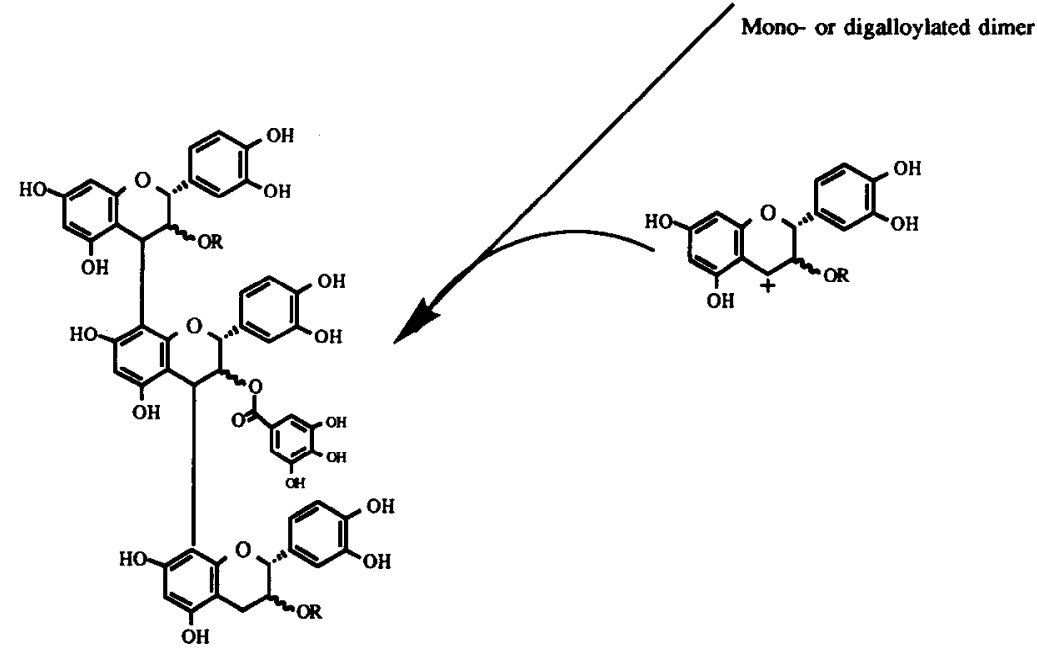

Mono- or digalloylated trimer

Fig. 2. Proposal for the synthesis of gallic esters of procyanidins. 
Another alternative to account for the synthesis of galloylated procyanidins, and explain the fact that no trimers esterified on their upper parts were found, would be to assume that the incorporation of the acyl group to a molecule of procyanidin occurs sequentially, the upper subunits being the last to be esterified. In this sense, if it is taken into account that very low levels of monogalloylated trimers and even lower ones of digalloylated trimers have been found, it would be logical to assume that the concentrations of the corresponding triester derivatives, if they existed, would be even lower, explaining why they were not detected. However, the existence of appreciable amounts of the dimer B2-3-O-gallate, in which the lower subunit is not galloylated, would be in disagreement with sequential galloylation starting from the lower subunit and would make the earlier hypothesis more feasible.

\section{ACKNOWLEDGEMENTS}

The authors are grateful to the Junta de Castilla y León (Grant No. 0907/90) and C.I.C.Y.T. (ref. ALI900983) for financial support of this work. Author Escribano-Bailón is indebted to D.G.I.C.Y.T. for a fellowship (ref. AP90). Thanks are also due to Nick Skinner for translation of this paper.

\section{REFERENCES}

Bourzeix, M., Weyland, D. \& Heredia, N. (1986). Etude des catéchines et des procyanidols de la grape de raisin, du vin et d'autres derivés de la vigne. Bull. OIV, 59, 1171-254.

Da Silva, J. M. R., Bourzeix, M., Cheynier, V. \& Moutounet,
M. (1991a). Procyanidin composition of Chardonnay, Mauzac and Grenache blanc grapes. Vitis, 30, 245-52.

Da Silva, J. M. R., Rigaud, J., Cheynier, V., Cheminat, A. \& Moutounet, M. (1991b). Procyanidin dimers and trimers from grape seeds. Phytochemistry, 30, 1259-64.

Da Silva, J. M. R., Rosec, J. P., Bourzeix, M., Mourgues, J. \& Moutounet, M. (1992). Dimer and trimer procyanidins in Carignan and Mourvédre grapes and red wines. Vitis, 31, 55-63.

Escribano-Bailón, M. T., Gutiérrez-Fernández, Y., RivasGonzalo, J. C. \& Santos-Buelga, C. (1992). Characterization of procyanidins of Vitis vinifera variety Tinta del País grape seeds. J. Agric. Food Chem., 40, 1794-9.

Ferreira, D., Steynberg, J. P., Burger, J. F. W. \& Bezuidenhoudt, B. C. B. (1992). In Rec. Adv. Phytochem., 26, 255.

Haslam, E., Opie, C. T. \& Porter, L. J. (1977). Procyanidin metabolism. A hypothesis. Phytochemistry, 16, 99-102.

Haslam, E. (1989). Plant Polyphenols. Vegetable Tannins Revisited. Cambridge University Press, Cambridge.

Heller, W. \& Forkmann, G. (1993). Biosynthesis of flavonoids. In The Flavonoids Advances in Research since 1986, ed. J. B. Harborne. Chapman \& Hall, London, p. 499.

Lea, A. G. H., Bridle, P., Timberlake, C. F. \& Singleton, V. L. (1979). The procyanidins of white grapes and wines. Am. J. Enol. Vitic., 30, 289-300.

Oszmianski, J. \& Sapis, J. C. (1989). Fractionation and identification of some low molecular weight grape seed phenolics. J. Agric. Food Chem., 37, 1293-7.

Romeyer, F. M., Macheix, J. \& Sapis, J. (1986). Changes and importance of oligomeric procyanidins during maturation of grape seeds. Phytochemistry, 25, 219-21.

Stafford, H. A. (1989). The enzymology of proanthocyanidin biosynthesis. In Chemistry and Significance of Condensed Tunnins, eds R. W. Hemingway \& J. J. Karchesy. Plenum Press, New York, p. 47.

Stafford, H. A. (1993). Proanthocyanidin and flavan-3-ol biosynthesis: comparison with the anthocyanidin pathway and future needs. In Polyphenolic Phenomena, ed. A. Scalbert. INRA, Paris, p. 73.

Stafford, H. A., Shimamoto, M. \& Lester, H. H. (1982). Incorporation of (14C) phenylalanine in to flavan-3-ols and procyanidins in cell suspension cultures of Douglas fir. Plant Physiol., 69, 1055-9. 